Pré-Publicações do Departamento de Matemática

Universidade de Coimbra

Preprint Number 05-04

\title{
DESCENT FOR PRIESTLEY SPACES
}

\author{
MARGARIDA DIAS AND MANUELA SOBRAL
}

\begin{abstract}
A characterization of descent morphism in the category of Priestley spaces, as well as necessary and sufficient conditions for such morphisms to be effective are given. For that we embed this category in suitable categories of preordered topological spaces were descent and effective morphisms are described using the monadic description of descent.
\end{abstract}

KEYWORDS: ordered (preordered) topological spaces, Priestley space, Stone space, regular and universal regular epimorphisms, (effective) descent morphisms, monadic categories.

AMS Subject Classification (2000): 18D30, 18A20, 18C20.

\section{Introduction}

A preordered topological space (a preordered space) is a triple $(X, \tau, \leq)$ where $X$ is a set, $\tau$ is a topology and $\leq$ is a preorder (i.e. a reflexive and transitive relation) on $X$. When $\leq$ is also antisymmetric, then $(X, \tau, \leq)$ is called an ordered topological space (an ordered space). They are the objects of the category $\mathcal{T}$ opPreord $(\mathcal{T} o p O r d)$ whose morphisms are the continuous maps which preserve the preorder (the order, respectively).

An ordered space $(X, \tau, \leq)$ is said to be totally order-disconnected if given $x \not \leq x^{\prime}$ in $X$ there exists a closed and open (clopen, for short) decreasing subset $U$ of $X$ such that $x^{\prime} \in U$ and $x \notin X$. The compact totally orderdisconnected spaces are called the Priestley spaces. The full subcategory of TopOrd whose objects are the Priestley spaces will be denoted by $\mathcal{P} s p$.

The category $\mathcal{P} s p$ is dually equivalent to the category of bounded distributive lattices $\mathcal{D}$ Lat, the well-known Priestley duality. Since $\mathcal{D}$ Lat is monadic over Set, it is easy to describe descent there with respect to the codomain fibration $\mathcal{D} L a t^{2} \rightarrow \mathcal{D}$ Lat: the effective descent morphisms are the descent morphisms and they are exactly the regular epimorphisms. Therefore, we conclude that, in $\mathcal{P} s p$, the classes of effective codescent morphisms, of codescent morphisms and of regular monomorphisms coincide.

Received March 24, 2005.

The authors gratefully acknowledge financial support from FCT/Centro de Matemática da Universidade de Coimbra. 
The descent morphisms with respect to the codomain fibration $\mathcal{P} s p^{2} \rightarrow \mathcal{P} s p$ form a class which is strictly contained in the one of the regular epimorphisms. Also the class of effective descent morphisms in $\mathcal{P} s p$ is a proper subclass of the one of the descent morphisms. To prove that we consider StonePreord, the full subcategory of $\mathcal{T}$ opPreord with objects all $(X, \tau, \leq)$ such that $(X, \tau)$ is a Stone space, as well as its full subcategory P Preord with objects all totally preordered-disconnected Stone spaces, and study the reflective embeddings of $\mathcal{P}_{s p}$ in PPreord and of PPreord in StonePreord.

Necessary and sufficient conditions for descent morphisms in PPreord and in StonePreord to be effective are obtained by embedding these categories into the category StoneRel, with objects all triples $\left(X, \tau, R_{X}\right)$ where $(X, \tau)$ is a Stone space and $R_{X}$ is an arbitrary binary relation on $X$. An explicit description of the effective descent morphisms in StonePreord is given, using the one presented in [3] for the effective descent morphisms in Preord. Finally, we prove that a $\mathcal{P} s p$-morphism is an effective descent morphism in $\mathcal{P} s p$ if and only if it is an effective descent morphism in $\mathcal{P}$ Preord.

For a comprehensive description of descent theory see [5].

\section{Fundamentals of Monadic Descent}

Let $\mathcal{C}$ be a category with pullbacks. The fibres with respect to the codomain functor $\mathcal{C}^{2} \rightarrow \mathcal{C}$ are the slice categories $\mathcal{C} \downarrow B$, for each $B \in \mathcal{C}$

For every $\mathcal{C}$-morphism $p: E \rightarrow B$, the pullback functor $p^{*}: \mathcal{C} \downarrow B \rightarrow \mathcal{C} \downarrow E$ has a left adjoint $p$ ! which is defined by composition with $p$ on the left.

For bifibrations satisfying Beck-Chevalley condition, descent data can be interpreted as structure maps for a monad, a fact first proved by Bénabou and Roubaud in [1]. This is the case of the bifibration above: the category Des $(p)$, of objects equipped with descent data and morphisms preserving it, is equivalent to the the category $(\mathcal{C} \downarrow E)^{\mathbb{T}}$ of $\mathbb{T}$-algebras for the monad $\mathbb{T}$ induced in $\mathcal{C} \downarrow E$ by the adjunction $p ! \dashv p^{*}(\eta, \epsilon)$.

Let $\Phi: \mathcal{C} \downarrow B \rightarrow(\mathcal{C} \downarrow E)^{\mathbb{T}}$ be the Eilenberg-Moore comparison functor. A morphism $p: E \rightarrow B$ is a descent morphism if $\Phi$ is full and faithful and it is an effective descent morphism if $\Phi$ is an equivalence of categories.

Proposition 1.1. For the monad $\mathbb{T}$ induced by $p ! \dashv p^{*}(\eta, \varepsilon)$ in $\mathcal{C} \downarrow E$ we have that:

(i) $\Phi$ is full and faithful if and only if $\varepsilon$ is pointwise a regular epimorphism. 
(ii) $\Phi$ has a left adjoint if and only if, for each $\mathbb{T}$-algebra $(C, \gamma: C \rightarrow$ $\left.E, \xi: E \times{ }_{B} C \rightarrow C\right), \mathcal{C} \downarrow B$ has coequalizers of the pair $\left(\pi_{2}, \xi\right)$.

A morphism $p$ is called a universal regular epimorphism if its pullback along any morphism is a regular epimorphism.

For each $(A, \alpha)$ in $\mathcal{C} \downarrow B, \varepsilon_{(A, \alpha)}=\pi_{2}:\left(E \times_{B} A, p \cdot \pi_{1}\right) \rightarrow(A, \alpha)$, where the diagram

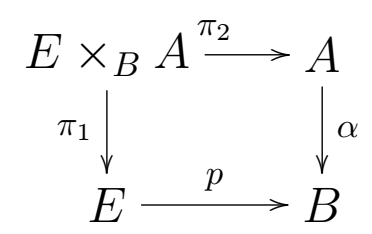

is the pullback of $p$ along $\alpha$. Then the following is an immediate consequence of 1.1(i):

Corollary 1.2. A morphism $p$ is a descent morphism if and only if it is a universal regular epimorphism.

We are going to describe descent in categories $\mathcal{C}$ that, other than pullbacks, have all coequalizers. Then $\mathcal{C} \downarrow B$ has coequalizers and they are constructed at the level of $\mathcal{C}$. Therefore, the corresponding comparison functor is always part of an adjunction $L \dashv \Phi(\alpha, \beta)$.

We first look for a characterization of the universal regular epimorphism in $\mathcal{C}$ because the following holds:

$p$ descent morphism $\Longleftrightarrow \beta$ is an isomorphism $\Longleftrightarrow p$ is a universal regular epimorphism.

For a $\mathbb{T}$-algebra $(C, \gamma, \xi)$ we consider the diagram

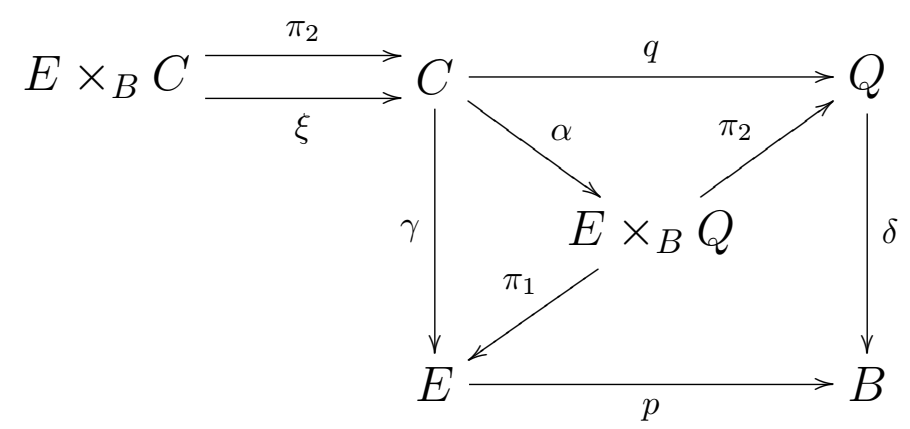

where $q$ is the coequalizer of the pair $\left(\pi_{2}, \xi\right)$ and $\alpha=\alpha_{(C, \gamma, \xi)}$, the component of the unit of the comparison adjunction, is the unique morphism for which the upper triangles commute. 
Proposition 1.3. Let $\mathcal{C}$ be a concrete category over Set with pullbacks and coequalizers which are preserved by the forgetful functor. Then $\alpha_{(C, \gamma, \xi)}$ is a bimorphism for every $\mathbb{T}$-algebra.

Proof: If $\alpha(c)=(\gamma(c), q(c))=\left(\gamma\left(c^{\prime}\right), q\left(c^{\prime}\right)=\alpha\left(c^{\prime}\right)\right.$ then $\gamma(c)=\gamma\left(c^{\prime}\right)$ and $q(c)=q\left(c^{\prime}\right)$. Since (see e.g. 5.A in [4])

$$
q(c)=q\left(c^{\prime}\right) \Longleftrightarrow \xi\left(\gamma(c), c^{\prime}\right)=c \Longleftrightarrow \xi\left(\gamma\left(c^{\prime}\right), c\right)=c^{\prime}
$$

and $\xi(\gamma(c), c)=c$ then

$$
c=\xi(\gamma(c), c)=\xi\left(\gamma\left(c^{\prime}\right), c\right)=c^{\prime} .
$$

Hence $\alpha_{(C, \gamma, \xi)}$ is an injective map.

Since $\alpha_{(C, \gamma, \xi)} \cdot \xi=p^{*}(q)$ and $p^{*}(q)=1 \times_{B} q$ is surjective, we conclude that $\alpha_{(C, \gamma, \xi)}$ is an epimorphism.

Corollary 2.8 in [6] holds for these categories, as observed there, giving a criterion that will be useful in the sequel.

Theorem 1.4. Let $\mathcal{C}$ be a concrete category over Set with coequalizers and pullbacks. If pullbacks are preserved by the forgetful functor then, for a morphism $p$, the following are equivalent:

(i) $p$ is an effective descent morphism;

(ii) for each $\mathbb{T}$-algebra $(C, \gamma, \xi)$ the coequalizer of $\left(\pi_{2}, \xi\right)$ is a universal regular epimorphism;

(iii) for each $\mathbb{T}$-algebra $(C, \gamma, \xi)$ the square in diagram (1) is a pullback.

This follows from general results (see [4] and [6]). They are presented here in the appropriated form for the context we are interested in.

\section{Regular and universal regular epimorphisms}

Let Preord be the category with objects $X=(X, \leq)$ where $X$ is a set and $\leq$ is a preorder on $X$ and with morphisms the preorder preserving maps. We denote by $R_{X}$ the subset $\left\{\left(x, x^{\prime}\right) \mid x \leq x^{\prime}\right\}$ of $X \times X$.

Proposition 2.1. Let $f: X \rightarrow Y$ be a morphism in Preord.

(i) $f$ is a regular epimorphism if and only if $f(X)=Y$ and $R_{Y}$ is the transitive closure of $f \times f\left(R_{X}\right)$;

(ii) $f$ is a universal regular epimorphism if and only if $R_{Y}=f \times f\left(R_{X}\right)$. 
For details see 2.2 and 2.3 in [3].

Let $\mathcal{C}$ Preord be the full subcategory of TopPreord with objects $(X, \tau, \leq)$ such that $(X, \tau)$ belongs to a full subcategory $\mathcal{C}$ of $\mathcal{T}$ op closed under pullbacks and finite subspaces. Then the forgetful functors $U: \mathcal{C}$ Preord $\rightarrow \mathcal{C}$ and $V:$ CPreord $\rightarrow$ Preord preserve pullbacks.

Proposition 2.2. A morphism $f$ in Preord is a (universal) regular epimorphism if and only if its underlying maps in $\mathcal{C}$ and in $\mathcal{P}$ reord are (universal) regular epimorphisms.

Proof: Let $f: X \rightarrow Y$ be a morphism in CPreord and $\left(\pi_{1}, \pi_{2}\right)$ be its kernel pair.

If $f$ is a regular epimorphism then the coequalizer diagram

$$
X \times_{Y} X \underset{\pi_{2}}{\longrightarrow} X \stackrel{\pi_{1}}{\longrightarrow} Y
$$

is preserved by the forgetful functor $U: \mathcal{C}$ Preord $\rightarrow \mathcal{C}$ because $U$ has both a left and a right adjoint defined on objects by $F(C)=\left(C, \Delta_{C}\right)$ and $G(C)=$ $(C, C \times C)$, respectively. Furthermore, assuming that $R_{Y}$ strictly contains the transitive closure of $f \times f\left(R_{X}\right)$, let $Y^{\prime}$ have the same underlying topological space as $Y$ and $R_{Y^{\prime}}$ be the transitive closure of $f \times f\left(R_{X}\right)$. The morphism $f^{\prime}: X \rightarrow Y^{\prime}$, defined by $f^{\prime}(x)=f(x)$ for each $x \in X$, is such that $f^{\prime} \cdot \pi_{1}=$ $f^{\prime} \cdot \pi_{2}$ but does not factor through $f$. Consequently, $f$ would not be the coequalizer of $\left(\pi_{1}, \pi_{2}\right)$ in CPreord.

Conversely, if the underlying morphisms of $\mathrm{f}$ in $\mathrm{C}$ and in Preord are regular epimorphisms and $g \cdot \pi_{1}=g \cdot \pi_{2}$ in CPreord, then both structures produce a unique factorization of $g$ through $f$, say $h$ and $h^{\prime}$ and, since $f$ is surjective we have that $h=h^{\prime}$ is the unique morphism $h$ in CPreord such that $h \cdot f=g$.

If $f$ is a universal regular epimorphism in $\mathcal{C}$ and in Preord then, as every pullback in CPreord

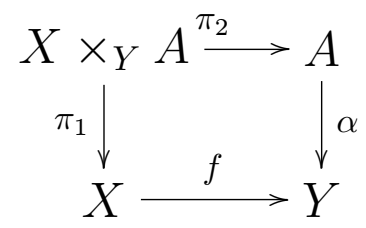

is preserved by $U$ and by $V, U\left(\pi_{2}\right)$ and $V\left(\pi_{2}\right)$ are regular epimorphisms and so $\pi_{2}$ is a regular epimorphism in ePreord. 
Let us assume now that $f$ is a universal regular epimorphism in CPreord. Then $U f$ is a universal regular epimorphism in $\mathcal{C}$ because the pullback of $U f$ along a morphism $\alpha^{\prime}: C \rightarrow U(Y)$ in $\mathcal{C}$ is the image by $U$ of a pullback in ePreord where $A=\left(C, \Delta_{C}\right)$.

For $y \leq y^{\prime}$ in $Y$, the pullback of $V(f)$ along $\alpha^{\prime}:\left\{y \leq y^{\prime}\right\} \rightarrow V(Y)$ is the image by $V$ of the pullback in ePreord of $f$ and $\alpha: A \rightarrow Y$ where $A$ is the ordered set $\left\{y \leq y^{\prime}\right\}$ with the subspace topology. Then $\pi_{2}$ is a regular epimorphism in CPreord and so in Preord. Since $X \times_{Y} A=f^{-1}(y) \times\{y\} \cup$ $f^{-1}\left(y^{\prime}\right) \times\left\{y^{\prime}\right\}$, there exist $(x, y) \leq\left(x^{\prime}, y^{\prime}\right)$ in $X \times_{Y} A$ which gives $x \leq x^{\prime}$ such that $f(x)=y$ and $f\left(x^{\prime}\right)=y^{\prime}$. Thus $V(f)$ is a universal regular epimorphism in Preord.

Proposition 2.3. Let $f: X \rightarrow Y$ be a morphism in StonePreord.

(i) $f$ is a regular epimorphism if and only if $f(X)=Y$ and $R_{Y}$ is the transitive closure of $f \times f\left(R_{X}\right)$;

(ii) $f$ is a universal regular epimorphism if and only if $R_{Y}=f \times f\left(R_{X}\right)$.

Proof: (i) and (ii) follow from the previous proposition and the fact that in the category Stone of Stone spaces the regular epimorphisms are universal and they are the surjective maps.

The category StonePreord has a factorization system $(\mathcal{E}, \mathcal{M})$ with $\mathcal{E}$ the class of regular epimorphisms and $\mathcal{M}$ the class of monomorphisms. Indeed,the $(\mathcal{E}, \mathcal{M})$-factorization of a morphism $f: X \rightarrow Y$ is obtained by considering the (RegularEpi, Mono)-factorization $f=m \cdot q$ in Stone, and endowing the codomain of $q$ with the preorder which is the transitive closure of $q \times q\left(R_{X}\right)$.

Proposition 2.4. The category PPreord is an epireflective subcategory of StonePreord.

Proof: For $(X, \tau, \leq)$ in StonePreord let $I(X)$ be $\left(X, \tau, \leq^{1}\right)$ with $x \leq^{1} x^{\prime}$ if

$$
\begin{aligned}
& x \leq x^{\prime} \text { in } X \text { or } \\
& x^{\prime} \in U \in D C \operatorname{Dopen}(X) \Longrightarrow x \in U,
\end{aligned}
$$

where DClopen $(X)$ denotes the set of decreasing clopen subsets of $X$. Then $\leq^{1}$ is a preorder on $I(X)$ which is an object of P Preord. The morphism $r_{X}: X \rightarrow I(X)$ defined by $r_{X}(x)=x$ is the reflection of $X$ in PPreord as we show next. Given $g: X \rightarrow Y$ with $Y \in \mathcal{P}$ Preord the unique continuous function $g^{\prime}: I(X) \rightarrow Y$ such that $g^{\prime} \cdot r_{X}=g$ is a preorder preserving map. Indeed, if $x \not x^{\prime}, x \leq^{1} x^{\prime}$ and $g(x) \not \leq g\left(x^{\prime}\right)$ there exists $U \in \operatorname{DClopen}(Y)$ 
which contains $g\left(x^{\prime}\right)$ but not $g(x)$. Hence, $x^{\prime} \in g^{-1}(U)$ which is a decreasing clopen subset of $X$ and $x \notin g^{-1}(U)$, a contradiction.

Furthermore, for each $X, r_{X}$, being a surjective map, is an epimorphism.

Proposition 2.5. Let $f: X \rightarrow Y$ be a morphism in PPreord and $\left(\pi_{1}, \pi_{2}\right)$ its kernel pair.

(i) $f$ is a regular epimorphism if and only if, up to isomorphism, $f=r_{Y^{\prime}} \cdot q$ where $q: X \rightarrow Y^{\prime}$ is the coequalizer of $\left(\pi_{1}, \pi_{2}\right)$ in StonePreord and $r_{Y^{\prime}}$ is the reflection of $Y^{\prime}$ in P Preord.

(ii) $f$ is an universal regular epimorphism if and only if $R_{Y}=f \times f\left(R_{X}\right)$.

Proof: (i) follows from the way colimits are constructed in full replete reflective subcategories of categories where these colimits exist.

(ii) The "if" part is clear. We prove the "only if" part. If $y \leq y^{\prime}$ in $Y$ let $A=\left\{y, y^{\prime}\right\}$ be the subspace of $Y$ in PPreord and consider the pullback along the inclusion $i: A \rightarrow Y$. Then, since $\pi_{2}$ is a regular epimorphism, $\pi_{2}=r_{A^{\prime}} \cdot q$.

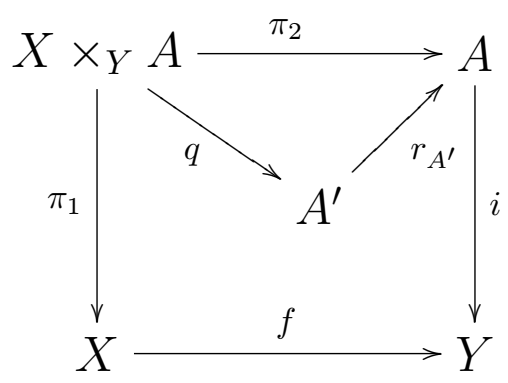

But finite discrete spaces are compact and totally preordered-disconnected for every preorder. Hence $A^{\prime}$ belongs to P Preord and so $r_{A^{\prime}}$ is an isomorphism. Now, like in the proof of 2.2 , we conclude that there exist $x \leq x^{\prime}$ in $X$ such that $f(x)=y$ and $f\left(x^{\prime}\right)=y^{\prime}$.

The category $\mathcal{P}$ Preord also has a factorization system $(\mathcal{E}, \mathcal{M})$ with $\mathcal{E}$ is the class of regular epimorphisms and $\mathcal{M}$ is the class of monomorphisms where the factorization of a morphism $f \in \mathcal{P}$ Preord is obtained by first considering the factorization $f=m \cdot q$ in StonePreord and then taking $f=m^{\prime} \cdot r_{Q} \cdot q$ 


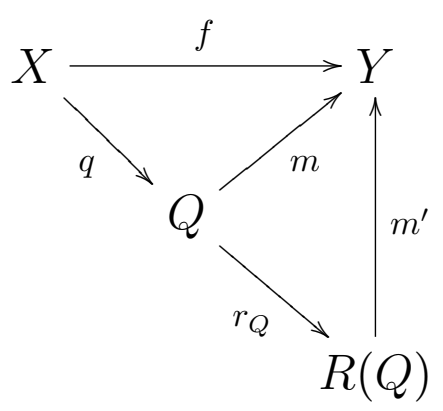

with $m^{\prime}$ the unique morphism such that $m^{\prime} \cdot r_{Q}=m$. Then $m^{\prime}$ is a monomorphism, since it is injective map, and $r_{Q} \cdot q$ is a coequalizer in PPreord.

Proposition 2.6. $\mathcal{P} s p$ is a regular-epireflective subcategory of $\mathcal{P}$ Preord.

Proof: For $X=(X, \tau, \leq) \in \mathcal{P}$ Preord we consider the binary relation

$$
x \sim x^{\prime} \text { if } x \leq x^{\prime} \text { and } x^{\prime} \leq x
$$

which is an equivalence relation on $X$.

Let $I(X)$ be the quotient set, $X / \sim$, equipped with the quotient topology with respect to the canonical projection $r_{X}: X \rightarrow I(X)$ and the preorder $R_{I(X)}$ obtained by transitive closure of $r_{X} \times r_{X}\left(R_{X}\right)$. Then we have that

$$
x \leq y \Leftrightarrow[x] \leq[y]
$$

where $[x]$ denotes the equivalence classe of $x$.

Being a continuous image of the compact space $X, I(X)$ is also compact. It remains to prove that it is totally order-disconnected. If $[x] \not \leq[y]$ then $x \not \leq y$ and so there exists a clopen decreasing subset $U_{1}$ of $X$ which contains $y$ but not $x$. Then the set $U=\left\{[a]: a \in U_{1}\right\}$ is a clopen subset of $X / \sim$, because $r_{X}^{-1}(U)=U_{1}$, and it is decreasing: if $[x] \leq[y] \in U$ then $x \leq y \in U_{1}$ which implies that $x \in U_{1}$ and so that $[x] \in U$. Thus $I(X)$ belongs to PSp.

Furthermore, given a morphism $g: X \rightarrow Y$ with $Y \in \mathcal{P} s p$ the unique function $g^{\prime}$ such that $g^{\prime} \cdot r_{X}=g$ is continuous and order preserving. Thus the regular epimorphism $r_{X}$ is the reflection of $X$ in $\mathcal{P}_{s p}$.

Proposition 2.7. Let $f: X \rightarrow Y$ be a morphism in $\mathcal{P} s p$.

(i) $f$ is a regular epimorphism in $\mathcal{P} s p$ if and only if it is a regular epimorphism in PPreord;

(ii) $f$ is a universal regular epimorphism in $\mathcal{P} s p$ if and only if $R_{Y}=$ $f \times f\left(R_{X}\right)$. 
Proof: (i) In categories with a system of factorization (RegularEpi, Mono) the embedding of each regular epireflective subcategory preserves and reflects regular epimorphisms.

(ii) In the proof of 2.5 (ii), $A^{\prime} \in \mathcal{P} s p$ and the proof that the condition is necessary follows in a completely analogous way.

Finally, being a regular epireflective subcategory of a category with a factorization system (RegularEpi, Mono), Psp also admits a (RegularEpi, Mono)factorization system.

\section{Descent in PPreord}

By Proposition 2.5(ii), a morphism $p: E \rightarrow B$ is a descent morphism in PPreord if and only if for each $b \leq b^{\prime}$ in $B$ there exist $e \leq e^{\prime}$ in $E$ such that $p(e)=b$ and $p\left(e^{\prime}\right)=b^{\prime}$, and these are the descent morphisms in PPreord as well as in the full subcategory FinPreord of finite preordered sets.

Proposition 3.1. (3.4, [3]) For a morphism $p: E \rightarrow B$ in Preord (or in FinPreord) the following are equivalent:

(i) $p$ is an effective descent morphism;

ii for every $b_{0} \leq b_{1} \leq b_{2}$ in $B$ there exists $e_{0} \leq e_{1} \leq e_{2}$ in $E$ such that $p\left(e_{i}\right)=b_{i}$, for $i=0,1,2$.

Proposition 3.2. (3.9, [4]) Let $\mathcal{D}$ be a full subcategory of $\mathcal{C}$ closed under pullback. If a morphism $p$ in $\mathcal{D}$ is an effective descent morphism in $\mathcal{C}$ then the following are equivalent:

(i) $p$ is an effective descent morphism in $\mathcal{D}$;

(ii) for every pullback

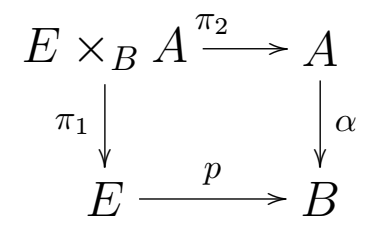

in $\mathcal{C}, A \in \mathcal{D}$ whenever $E \times_{B} A \in \mathcal{D}$.

Corollary 3.3. Let $\mathcal{D}$ be a full subcategory of $\mathcal{C}$ closed under pullbacks. If the descent morphisms in $\mathcal{D}$ are effective descent morphisms in $\mathcal{C}$ then the following are equivalent:

(i) $p$ is an effective descent morphisms in $\mathcal{D}$;

(ii) $p$ is a descent morphism in $\mathcal{D}$ and, for every pullback (2) in $\mathcal{C}, A \in \mathcal{D}$ whenever $E \times \times_{B} A \in \mathcal{D}$. 
Proposition 3.4. In StoneRel the effective descent morphisms are the regular epimorphisms.

Proof: The universal epimorphisms in Stone Rel are the morphisms whose underlying morphisms in Stone and in $\mathcal{R e l}$ are universal regular epimorphisms. Indeed, Proposition 2.2 is still true if instead of CPreord we consider CRel, the category of spaces of $\mathcal{C}$ equipped with an arbitrary binary relation. In the this case only the left adjoint to the forgetful functor $U: \mathcal{C} R e l \rightarrow \mathfrak{C}$, has a different definition: $F(C)=(C, \emptyset)$ for each $C \in \mathcal{C}$.

In Stone the regular epimorphisms are universal and they are exactly the surjective maps. In Rel a regular epimorphism is a morphism $f: X \rightarrow Y$ such that $f(X)=Y$ and $R_{Y}=f \times f\left(R_{X}\right)$ and so it is also a universal regular epimorphism. Consequently, every regular epimorphism in StoneRel is a universal regular epimorphism. Now the conclusion follows by applying Theorem 1.4.

Proposition 3.5. For a morphism $p: E \rightarrow B$ in P Preord the following are equivalent:

(i) $p$ is an effective descent morphism;

(ii) $p$ is a descent morphism and, for every pullback (2) in StoneRel, $A \in$ PPreord whenever $E \times_{B} A \in \mathcal{P}$ Preord.

Proof: We can apply Corollary 3.3 to $\mathcal{D}=$ PPreord and $\mathcal{C}=$ StoneRel. Indeed, PPreord is a full subcategory of StoneRel closed under pullbacks and every descent morphism in PPreord is an effective descent morphism in StoneRel.

We can also apply 3.3 to $\mathcal{D}=$ StonePreord and $\mathcal{C}=$ StoneRel. In this case this case one can give an explicit description of the effective descent morphisms in StonePreord.

Proposition 3.6. For a morphism $p: E \rightarrow B$ in StonePreord the following are equivalent:

(i) $p$ is an effective descent morphism;

(ii) $p$ is a descent morphism and, for every pullback (2) in StoneRel, $A \in$ StonePreord whenever $E \times_{B} A \in$ StonePreord

(iii) for every $b_{0} \leq b_{1} \leq b_{2}$ in $B$ there exists $e_{0} \leq e_{1} \leq e_{2}$ in $E$ such that $p\left(e_{i}\right)=b_{i}$, for $i=0,1,2$. 
Proof: By 3.4 in [3], that we recall in 3.1, (ii) $\Leftrightarrow$ (iii) tell us that the effective descent morphisms in StonePreord are exactly those morphisms whose underlying morphisms in Preord are the effective descent morphisms in this category. The proof given there still holds if we replace sets by Stone spaces as we sketch now.

If $p$ satisfies (ii) and $b_{0} \leq b_{1} \leq b_{2}$ in $B$ let $A$ be the set $\left\{a_{0}, a_{1}, a_{2}\right\}$ equipped with the binary relation $R_{A}=\left\{\left(a_{0}, a_{1}\right),\left(a_{1}, a_{2}\right)\right\} \cup \Delta_{A}$ and the discrete topology and $\alpha$ be defined by $\alpha\left(a_{i}\right)=b_{i}$ for $\mathrm{i}=0,1,2$. For the pullback (2) in StoneRel, since $E \times_{B} A \notin$ StonePreord, $R_{E \times_{B} A}$ is not transitive. Hence there exist $\left(x_{0}, x_{1}\right),\left(x_{1}, x_{2}\right) \in R_{E \times_{B} A}$ but not $\left(x_{1}, x_{2}\right)$. Since $\pi_{2}$ is a regular epimorphism in Stone Rel we can conclude that $x_{i}$ belongs to $p^{-1}\left(b_{i}\right) \times\left\{a_{i}\right\}$ and then that $e_{i}=\pi_{1}\left(x_{i}\right)$ form a chain $e_{0} \leq e_{1} \leq e_{2}$ in $E$ such that $p\left(e_{i}\right)=b_{i}$ for $\mathrm{i}=0,1,2$.

Conversely, it is easy to show that condition (iii) implies that $R_{A}$ is transitive when the relation $R_{E \times_{B} A}$ is transitive.

Proposition 3.7. A morphism $p: E \rightarrow B$ in PPreord is an effective descent morphism if:

(i) for every $b_{0} \leq b_{1} \leq b_{2}$ in $B$ there exists $e_{0} \leq e_{1} \leq e_{2}$ in $E$ such that $p\left(e_{i}\right)=b_{i}$, for $i=0,1,2$.

(ii) for every pullback (2) in StonePreord, if $E \times_{B} A$ belongs to P Preord then also $A$ belongs to PPreord.

Proof: Since $\mathcal{D}=\mathcal{P}$ Preord is a full subcategory of $\mathcal{C}=$ StonePreord closed under pullbacks, we apply 3.2 and the characterization above to conclude that these are sufficient conditions for a descent morphism in PPreord to be an effective descent morphism.

Condition (ii) above is necessary for a descent morphism in $\mathcal{P}$ Preord to be an effective descent morphism.

Proposition 3.8. If $p$ is an effective descent morphism in $\mathcal{P}$ Preord then for every pullback (2) in StonePreord, if $E \times_{B} A$ belongs to PPreord then also $A$ belongs to PPreord.

Proof: Given a pullback (2) in StonePreord with $E \times_{B} A \in \mathcal{P}$ Preord then we have that

- $\left(E \times_{B} A, \pi_{1}, \pi_{13}\right) \in(\text { P Preord } \downarrow E)^{\mathbb{T}}$;

- $\pi_{2}$ is a coequalizer of $\left(\pi_{23}, \pi_{13}\right)$ in StonePreord, because $p$ is a descent morphism; 
- $q=r_{A} \cdot \pi_{2}$ is the coequalizer of $\left(\pi_{23}, \pi_{13}\right)$ in PPreord.

By 1.4, $q$ is a universal regular epimorphism in PPreord. Then for $a \leq a^{\prime}$ in $I(A)$ there exist $(e, a) \leq\left(e^{\prime}, a^{\prime}\right) \in E \times_{B} A$ and so $a \leq a^{\prime}$ in $A$. Hence $r_{A}$ is an isomorphism and so that $A$ belongs to PPreord.

We can apply 3.2 to $\mathcal{D}=\mathcal{F}$ inP Preord, the subcategory of finite spaces in $\mathcal{P}$ Preord, which is isomorphic FinPreord, and $\mathcal{C}=\mathcal{P}$ Preord to conclude that a morphism $p \in \mathcal{D}$ which is an effective descent morphism in $\mathcal{P}$ Preord is also an effective descent morphism in $\mathcal{D}$. Then, the class of descent morphisms in $\mathcal{P}$ Preord strictly contains the one of the effective descent morphisms in this category since we also have strict inclusion of the corresponding classes in FinPreord.

\section{Descent in $\mathcal{P} s p$}

Let $H: X \rightarrow \mathcal{C}$ be the inclusion and $I$ the reflection of $\mathcal{C}$ in $\mathcal{X}$. Reflections that preserve pullbacks of all pairs with codomain in $\mathcal{X}$ are said to have stable units in [2].

Lemma 4.1. Let $\mathcal{C}, \mathcal{C}^{\prime}, X$ and $\mathcal{X}^{\prime}$ be categories with pullbacks. Given reflections $H \dashv I: \mathcal{C} \rightarrow X$ and $I^{\prime} \dashv H^{\prime}: \mathcal{X}^{\prime} \rightarrow \mathcal{C}^{\prime}$ and pullback preserving functors $U$ and $V$ for which the diagram

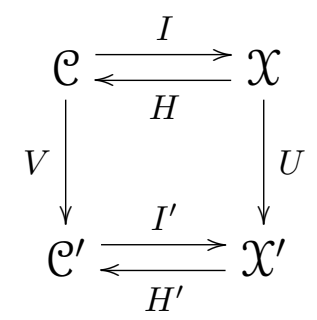

commutes, if $H^{\prime} \dashv I^{\prime}$ has stable units and $U$ reflects isomorphisms then $H \dashv I$ also has stable units.

Proposition 4.2. The reflection $H \dashv I:$ P Preord $\rightarrow \mathcal{P}$ sp has stable units.

Proof: Consider the following commutative diagram

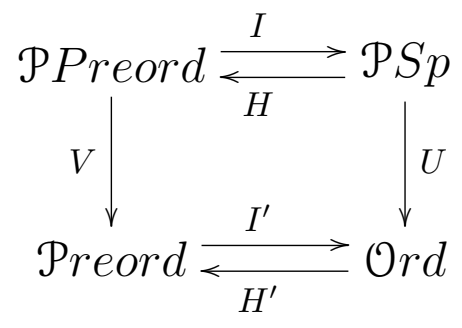


where the bottom is the reflection of Preord in $\mathcal{O r d}$, the category of (partially) ordered sets and $U, V$ are the obvious forgetful functors. In [7], J. Xarez proved that the reflection of Preord in $\mathcal{O} r d$ has stable units. Since $U$ and $V$ preserves pullbacks and $U$ reflects isomorphisms we conclude that the reflection of $\mathcal{P}$ Preord in $\mathcal{P} s p$ also has stable units.

Theorem 4.3. A morphism $p: E \rightarrow B$ in $\mathcal{P} s p$ is an effective descent morphism in this category if and only if it is an effective descent morphism in PPreord

Proof: Let $p: E \rightarrow B \in \mathcal{P}_{s p}$ be an effective descent morphism in PPreord. Since $\mathcal{P} s p$ is a full subcategory of $\mathcal{P}$ Preord closed under pullbacks, by 3.2, for a pullback

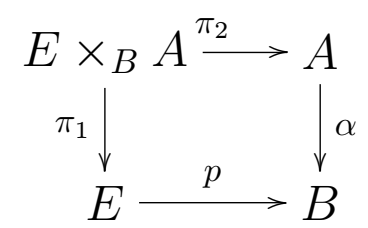

in PPreord with $E \times_{B} A \in \mathcal{P} s p$ we have to prove that $A \in \mathcal{P}$ Preord. Since the reflection $I: \mathcal{P}$ Preord $\rightarrow \mathcal{P} s p$ has stable units and $B \in \mathcal{P} s p$ then $I$ preserve the pullback. Thus, in the diagram

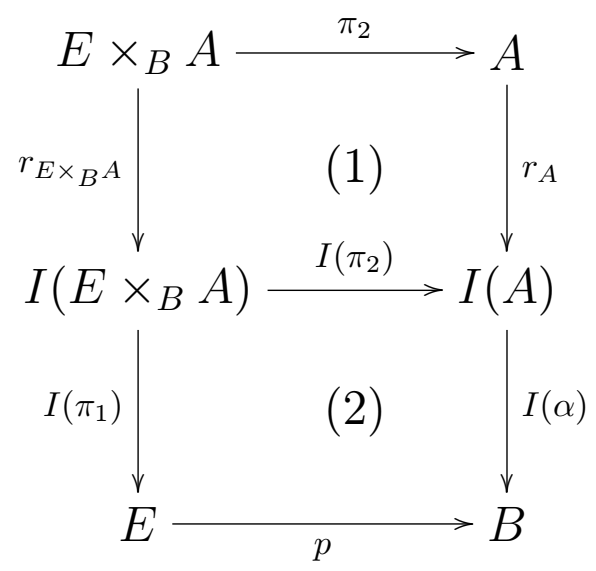

the outer rectangle $(1)+(2)$ as well as the square (2) are pullbacks. Consequently, (1) is a pullback and so, for the pullback functor $p^{*}: \mathcal{P}$ Preord $\downarrow$ $B \rightarrow \mathcal{P}$ Preord $\downarrow E, p^{*}\left(r_{A}\right)=r_{E \times_{B} A}$ which is an isomorphism because $E \times_{B} A$ belongs to Psp. But $p^{*}: \mathcal{P}$ Preord $\downarrow B \rightarrow$ P Preord $\downarrow E$, being monadic, reflects isomorphisms. Therefore, $r_{A}$ is an isomorphism and so $A$ belongs to Psp.

Conversely, if the morphism $p$ is an effective descent morphism in $\mathcal{P} s p$ it is a descent morphism also in $\mathcal{P}$ Preord. Indeed, let $(C, \gamma, \xi)$ be a $\mathbb{T}$-algebra for 
the monad induced in $\mathcal{P}$ Preord $\downarrow E$ by the adjunction $p$ ! $\dashv p^{*}: \mathcal{P}$ Preord $\downarrow$ $B \rightarrow$ PPreord $\downarrow E$.

Since $I$ preserves pullbacks of morphisms with codomain in $\mathcal{P} s p$ it is easy to see that $(I(C), I(\gamma), I(\xi))$ is a $\mathbb{T}$-algebra for the monad induced in $\mathcal{P} s p \downarrow E$ by the adjunction $p ! \dashv p^{*}: \mathcal{P}_{s p} \downarrow B \rightarrow \mathcal{P}_{s p} \downarrow E$.

In the diagram

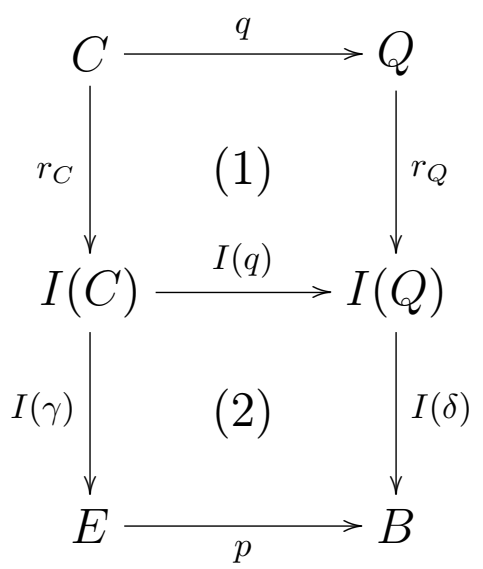

the square (2) is a pullback: since $p$ is an effective descent morphism in $\mathcal{P} s p$ and the forgetful functor from $\mathcal{P} s p$ to $\mathcal{S}$ et preserves pullbacks and coequalizers the conclusion follows from 1.4.

We can apply 1.4 to $\mathcal{C}=\mathcal{P}$ Preord and prove that the outer rectangle (1) $+(2)$ is a pullback, which is equivalent to prove that (1) is a pullback.

Let $h: C \rightarrow I(C) \times_{I(Q)} Q$ be the morphism defined by $h(c)=([c], q(c))$. There exists an isomorphism $t: E \times_{B} Q \rightarrow I(C) \times_{I(Q)} Q$ such that $h=$ $t \cdot \alpha_{(C, \gamma, \xi)}$. Then, by 1.3 , we conclude that $h$ is a bijective map and so an homeomorphism.

Furthermore it is an order isomorphism: if $h(c) \leq h\left(c^{\prime}\right)$ then $[c] \leq\left[c^{\prime}\right]$ and so $c \leq c^{\prime}$ in $C$. Thus $h$ is an isomorphism in PPreord and so $p$ is an effective descent morphism in $\mathcal{P}$ Preord.

\section{References}

[1] J. Bénabou and J. Roubaud, Monades et descente, Comptes Rendus Acad. Sc. Paris 270 A (1970), 96-98.

[2] C. Cassidy, M. Hébert and G. M. Kelly, Reflective subcategories, localization and factorization systems, J. Austral. Math. Soc. (Ser. A) 38(1985), 287-329.

[3] G. Janelidze and M. Sobral, Finite preorders and topological descent I, J. Pure Appl. Algebra 175 (2002), 187-205.

[4] G. Janelidze, M. Sobral and W. Tholen, Beyond Barr Exactness: Effective Descent Morphisms, in Categorical Foundations. Special Topics in Order, Topology, Algebra and Sheaf Theory, Cambridge University Press, 2004. 
[5] G. Janelidze and W. Tholen, Facets of descent I, Applied Cat. Struct. 2 (1994), 245-281.

[6] M. Sobral and W. Tholen, Effective descent morphisms and effective equivalence relations, Conference Proceedings of the Canadian Mathematical Society 13(1992), 421-433.

[7] J. Xarez, The monotone-light factorization for categories via preordered and ordered sets, PhD Thesis, Universidade de Aveiro (Portugal), 2003.

MARGARIDA DIAS

Departamento de Matemática, Universidade dos AÇOres, 9501-801 Ponta Delgada, PorTUGAL

E-mail address: mdias@notes.uac.pt

MANuEla SOBRAL

Departamento de Matemática, Universidade de Coimbra, 3001-454 Coimbra, Portugal

E-mail address: sobral@mat.uc.pt 